\title{
Scaling of the elastic contribution to the surface free energy of a nematic liquid crystal on a sawtoothed substrate
}

\author{
Jose Manuel Romero-Enrique, ${ }^{1}$ Chi-Tuong Pham, ${ }^{2}$ and Pedro Patrício ${ }^{3,4}$ \\ ${ }^{1}$ Departamento de Física Atómica, Molecular y Nuclear, Area de Física Teórica, Universidad de Sevilla, \\ Apartado de Correos 1065, 41080 Sevilla, Spain \\ ${ }^{2}$ Laboratoire d'Informatique pour la Mécanique et les Sciences de l'Ingénieur, CNRS-UPR 3251, \\ Université Paris-Sud 11, BP 133, F-91403 Orsay Cedex, France \\ ${ }^{3}$ Instituto Superior de Engenharia de Lisboa, Rua Conselheiro Emídio Navarro 1, P-1949-014 Lisboa, Portugal \\ ${ }^{4}$ Centro de Física Teórica e Computacional, Universidade de Lisboa, Avenida Professor Gama Pinto 2, \\ P-1649-003 Lisboa Codex, Portugal
}

(Received 7 October 2009; published 16 July 2010)

\begin{abstract}
We characterize the elastic contribution to the surface free energy of a nematic liquid crystal in the presence of a sawtooth substrate. Our findings are based on numerical minimization of the Landau-de Gennes model and analytical calculations on the Frank-Oseen theory. The nucleation of disclination lines (characterized by nonhalf-integer winding numbers) in the wedges and apexes of the substrate induces a leading order proportional to $q \ln q$ to the elastic contribution to the surface free-energy density, with $q$ being the wave number associated with the substrate periodicity.
\end{abstract}

DOI: 10.1103/PhysRevE.82.011707

PACS number(s): $61.30 .-\mathrm{v}$

\section{INTRODUCTION}

Topological defects are ubiquitous in many branches of physics, spanning from condensed-matter physics $[1,2]$ to cosmology [3]. They may emerge in systems with broken continuous symmetry, and their presence can introduce essential singularities in the free energy which lead to infiniteorder phase transitions such as the Kosterlitz-Thouless transition $[4,5]$. Liquid-crystalline phases, such as nematics, are prototypical for showing topological defects, such as point hedgehogs and disclination lines [6,7]. Topological arguments show that within the bulk and in three dimensions the only stable disclination lines have $\pm 1 / 2$ winding number [2]. The presence of substrates may induce the nucleation of disclinations with other winding numbers. Theoretically, non-half-integer disclination lines are predicted to nucleate in the cusps (wedges and/or apexes) of substrates [8-11], as well as in sharp boundaries between domains characterized by different anchoring conditions in flat surfaces $[12,13]$. Furthermore, there is some experimental evidence of the formation of these unusual non-half-integer disclination lines on surfaces [14].

It is well known that the nematic director field in the presence of structured substrates may be distorted, leading to an elastic contribution to the free energy. Since the seminal work by Berreman $[15,16]$, this problem has been extensively studied and generalized in the literature [8-11,17-22]. However, most of these studies focused on smooth substrates or on the effect of isolated cusps. In this paper we will consider the effect that the disclination lines nucleating on the cups of the substrates have on the elastic contribution to the surface free energy of the nematic on periodic and cusped structures. In particular, we will consider a sawtoothed substrate which favors homeotropic anchoring. Other geometries may also be considered with our formalism, such as steplike substrates, which have been studied numerically and experimentally in the context of zenithal bistable switching in nem- atic devices $[17,23,24]$. We will show that the disclination lines induce a contribution to the elastic contribution to the free-energy density (per unit projected area) which depends only on the geometric characteristics of the substrate, and that scales with the wave number $q$ associated with the periodicity of the substrate as $-q \ln q$

The paper is organized as follows. The different models for the nematic in the presence of a sawtooth substrate are presented in Sec. II. We focus in particular on substrates which favor homeotropic anchoring and their consequences. In Sec. III we report the numerical and analytical results obtained from these approaches. In Sec. IV we present our conclusions.

\section{MODEL}

We consider a nematic phase in contact with a sawtooth substrate characterized by an angle $\alpha$ and a side length $L$ (see Fig. 1). At the substrate the nematic molecules preferentially align homeotropically, i.e., parallel to the local normal to the substrate. The system is translationally invariant along the

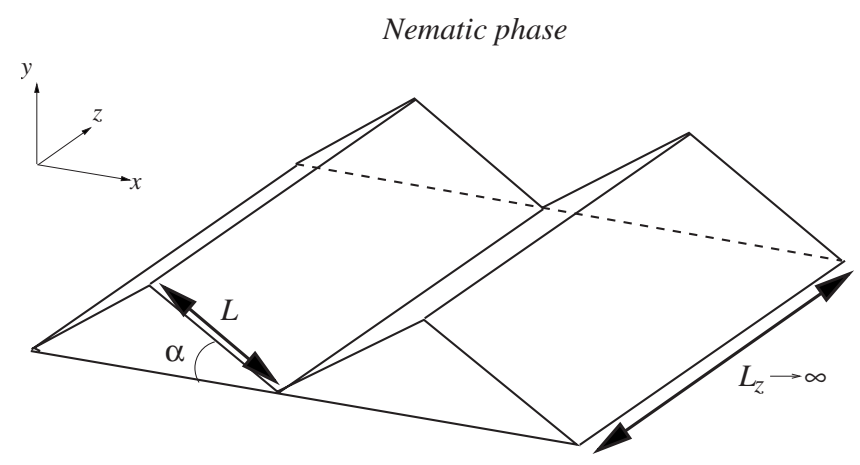

FIG. 1. Schematic picture of the geometry of the system, characterized by the side length $L$ and the angle $\alpha$. 
out-of-plane axis $z$ and periodic along the $x$ axis. We will only consider azimuthal distortions for the nematic director $\mathbf{n}$, which can be parametrized in terms of the angle $\theta$ between the director and the $y$ direction as $\mathbf{n}=(-\sin \theta, \cos \theta, 0)$. Out-of-plane or twist deformations may also be important under other conditions, as a twist instability may occur [25], but we checked numerically that they are not relevant for our choice of parameters as free-energy minimization always leads to azimuthal distorted textures. Finally, we impose that, far from the substrate, the bulk nematic phase orients homogeneously along either the $x$ (the $N^{\|}$ texture) or the $y$ (the $N^{\perp}$ texture) direction, which are the only relevant situations allowed by symmetry considerations. We study the orientational ordering of this system within two different models: the Landau-de Gennes (LdG) model and the Frank-Oseen (FO) model.

\section{A. Landau-de Gennes model}

In the LdG model, both isotropic and nematic phases can be locally represented by a traceless symmetric order parameter tensor with components $Q_{i j}$, which can be represented as $Q_{i j}=\frac{3}{2} S\left[n_{i} n_{j}-\frac{1}{3} \delta_{i j}\right]+\frac{1}{2} B\left[l_{i} l_{j}-m_{i} m_{j}\right]$, where $n_{i}$ are the Cartesian components of the director field $\mathbf{n}, S$ is the nematic order parameter which measures the orientational ordering along the nematic director, and $B$ is the biaxiality parameter, which measures the ordering of the molecules on the orientations perpendicular to $\mathbf{n}$, characterized by the eigenvectors $\mathbf{l}$ and $\mathbf{m}$. The LdG free energy can be written as $\mathcal{F}_{\mathrm{LdG}}=\int_{\mathcal{V}}\left(\phi_{\text {bulk }}+\phi_{\mathrm{el}}\right) d V+\int_{\mathcal{A}} \phi_{\text {surf }} d s$, where $\phi_{\text {bulk }}$ is the bulk free-energy density, $\phi_{\mathrm{el}}$ is the elastic free-energy density, and $\phi_{\text {surf }}$ is the surface free energy defined as [16]

$$
\begin{gathered}
\phi_{\text {bulk }}=a \operatorname{Tr} \mathbf{Q}^{2}-b \operatorname{Tr} \mathbf{Q}^{3}+c\left[\operatorname{Tr} \mathbf{Q}^{2}\right]^{2}, \\
\phi_{\mathrm{el}}=\frac{L_{1}}{2} \partial_{k} Q_{i j} \partial_{k} Q_{i j}+\frac{L_{2}}{2} \partial_{j} Q_{i j} \partial_{k} Q_{i k}, \\
\phi_{\text {surf }}=-\frac{2}{3} w \operatorname{Tr}\left[\mathbf{Q} \cdot \mathbf{Q}_{\text {surf }}\right],
\end{gathered}
$$

where $a$ depends linearly on the temperature, $b$ and $c$ are positive constants, and $L_{1}$ and $L_{2}$ are positive parameters related to the elastic constants. If we will rescale all the variables as follows [26]: $\tilde{\mathbf{Q}}=6 c \mathbf{Q} / b$, the positions $\tilde{\mathbf{r}}=\mathbf{r} / \xi$, where the correlation length $\xi$ is defined as $\xi^{2}=8 c\left(3 L_{1}+2 L_{2}\right) / b^{2}$, and $\tilde{\mathcal{F}}_{\mathrm{LdG}}=24^{2} c^{3} \mathcal{F}_{\mathrm{LdG}} / \xi^{3} b^{4}$, we get $\widetilde{\mathcal{F}}_{\mathrm{LdG}}=\int \tilde{\mathcal{V}}\left(\widetilde{\phi}_{\text {bulk }}+\widetilde{\phi}_{\mathrm{el}}\right) d \widetilde{V}+\int_{\tilde{\mathcal{A}}} \widetilde{\phi}_{\text {surf }} d \widetilde{s}$, with rescaled freeenergy densities

$$
\begin{gathered}
\widetilde{\phi}_{\text {bulk }}=\frac{2}{3} \tau \operatorname{Tr} \widetilde{\mathbf{Q}}^{2}-\frac{8}{3} \operatorname{Tr} \widetilde{\mathbf{Q}}^{3}+\frac{4}{9}\left[\operatorname{Tr} \widetilde{\mathbf{Q}}^{2}\right]^{2}, \\
\widetilde{\phi}_{\mathrm{el}}=\frac{1}{3+2 \kappa}\left[\widetilde{\partial}_{k} \widetilde{Q}_{i j} \widetilde{\partial}_{k} \widetilde{Q}_{i j}+\kappa \widetilde{\partial}_{j} \widetilde{Q}_{i j} \widetilde{\partial}_{k} \widetilde{Q}_{i k}\right],
\end{gathered}
$$

$$
\widetilde{\phi}_{\text {surf }}=-\frac{2}{3} \widetilde{w} \operatorname{Tr}\left[\tilde{\mathbf{Q}} \cdot \tilde{\mathbf{Q}}_{\text {surf }}\right] .
$$

Here, $\tau=24 a c / b^{2}$ is a dimensionless temperature, $\kappa=L_{2} / L_{1}$ is an elastic dimensionless parameter $(\kappa>-3 / 2)$ and $\widetilde{w}=16 w c / b^{2} \xi$ is the dimensionless anchoring strength. Hereafter we will consider these rescaled expressions, so we will drop the overtilde notation. For $\tau=1$, the bulk free-energy density has two minima corresponding to $\phi_{\text {bulk }}=0$ for rescaled scalar order parameters $S_{I}=0$ (isotropic phase) and $S_{N}=1$ (nematic phase), so both phases are at coexistence. It is important to note that the order parameter $S$ in the coexisting nematic phase is rescaled, so its value in real units is $b / 6 c$, which must be smaller than 1 (typically $\approx 0.4$ ). If the elastic parameter $\kappa$ is positive (negative), the nematic prefers to align parallel (perpendicular) to a possible nematicisotropic (NI) interface. Finally, $\mathbf{Q}_{\text {surf }}$ defines the favored tensor at the substrate. We will favor a homeotropic alignment of the nematic by setting $\mathbf{Q}_{\text {surf }}=(3 \boldsymbol{\nu} \otimes \boldsymbol{\nu}-1) / 2$, with $\boldsymbol{\nu}$ as the normal vector to the substrate, establishing a direct connection to previous papers [27-30]. Hereafter we will restrict ourselves to the nematic phase at the NI transition $(\tau=1)$, with $\kappa=2$.

\section{B. Frank-Oseen model}

The FO model can be considered as an approximation to the LdG model, in which we assume that the variations of the nematic order parameter are restricted to the neighborhood of the substrate (with a width on the order of $\xi$ ) and inside defect cores, and takes the bulk value $S$ elsewhere. Assuming that there are no disclination lines in the bulk, substitution of this Ansatz into the LdG free-energy functional leads to the following FO approach for large $L$ (in units of $\xi)$ :

$$
\mathcal{F} \approx \mathcal{F}_{\mathrm{FO}} \equiv \phi_{\text {bulk }}(S) \mathcal{V}+\int_{\mathcal{A}} d s \Sigma(\theta(s))+\frac{K}{2} \int_{\mathcal{V}} d V|\nabla \theta|^{2},
$$

where $\mathcal{V}$ is the total volume of the nematic, $\mathcal{A}$ is the substrate area in contact with the nematic, the elastic constant $K=(9 / 2) S^{2}(2+\kappa) /(3+2 \kappa)$ and $\Sigma(\theta)$ is an effective anchoring potential to be determined in the following that is due to the nematic order parameter distortions close to the substrates.

In order to make a quantitative comparison with the results within the LdG model, instead of considering an ad hoc expression for the anchoring potential, we will derive it from the LdG model. As it was mentioned above, the nematic order parameter distortions are assumed to be confined to a layer of width $\eta \sim \xi$ close to the substrates. On the other hand, the variations of the nematic director field close to the walls are rather small in directions parallel to the surfaces for large $L$ (except close to the wedges and apexes). So, we may estimate $\Sigma(\theta)$ as the LdG excess free energy per unit area of a slab of width $\eta$, for which, at the boundary $y=0$, we impose a surface field $\phi_{\text {surf }}$, and for $y \geq \eta$ we consider a bulk nematic phase with an uniform nematic director character- 


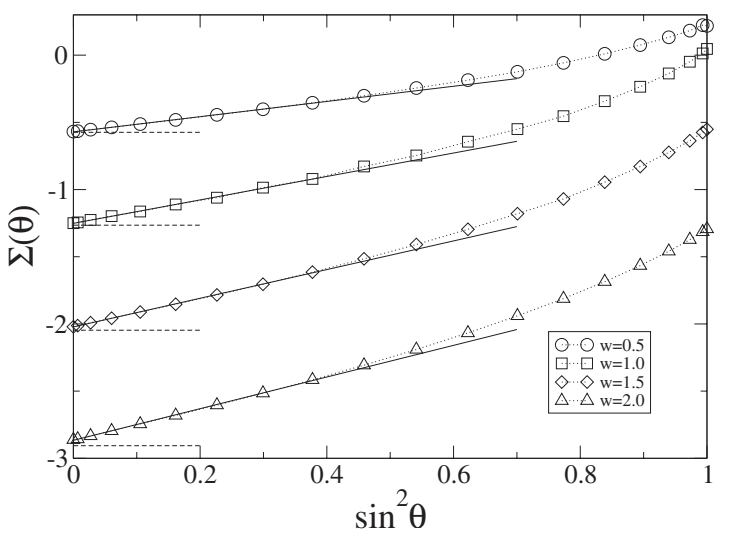

FIG. 2. Plot of the effective anchoring potential $\Sigma(\theta)$ as a function of $\sin ^{2} \theta$, for a layer width $\eta=1.5 \xi$ and $w=0.5$ (circles), $w=1$ (squares), $w=1.5$ (diamonds), and $w=2$ (triangles). The dashed horizontal lines correspond to the values of $\sigma_{\mathrm{NW}}$ obtained from Eq. (8), and the continuous lines correspond to the fit to a RapiniPapoular expression for small values of the tilt angle. The values of the slopes $\Sigma_{0}^{\prime \prime}$ are $0.565_{5}(w=0.5), 0.872_{9}(w=1), 1.067_{8}(w=1.5)$, and $1.177_{11}(w=2)$.

ized by a tilt angle $\theta$ with respect to the $y$ axis. Figure 2 shows $\Sigma(\theta)$ obtained by numerical minimization with a conjugate-gradient method for $\eta=1.5 \xi$ (similar results are obtained for other values of $\eta$ ). For each value of $w$, the minimum value of $\Sigma(\theta)$ corresponds to the homeotropic alignment $\theta=0$, where it takes the (true) nematic-wall surface tension value $\sigma_{\mathrm{NW}}(w)$. For not too large values of $\theta, \quad \Sigma(\theta)$ takes a Rapini-Papoular form [31] $\Sigma(\theta) \sim \sigma_{\mathrm{NW}}+\Sigma_{0}^{\prime \prime}(w) \sin ^{2} \theta$. There are deviations for $\theta$ around $\pi / 2$, but in any case the involved energies are much larger. In the range of values of $w$ we will consider in this paper $(0<w<2)$, both $\sigma_{\mathrm{NW}}$ and $\Sigma_{0}^{\prime \prime}$ are on the order of $w$. Indeed $\sigma_{\mathrm{NW}}$ can be obtained analytically from minimization of the LdG functional in the presence of a flat wall and for homeotropic anchoring [27,28]. In this situation the equilibrium nematic director field does not show any deformation and there is no biaxiality, so the resulting free energy at NI coexistence can be expressed in terms of the nematic order parameter profile $S=S(y)$ as $\mathcal{F}_{\mathrm{LdG}}=\mathcal{A} \int_{0}^{\infty}\left[S^{2}-2 S^{3}+S^{4}+\left(S^{\prime}\right)^{2} / 2\right] d y-w S(0)$. where $S^{\prime}(y)=d S / d y$. By introducing a magnetizationlike field $m(y) \equiv 2 S(y)-1$, the LdG functional reduces to the LandauGinzburg free-energy functional of an Ising model for zero applied magnetic field in the presence of a flat wall. This problem has been extensively studied in the literature $[32,33]$, leading to a nematic order parameter profile $S(y)=[1+g \exp (-\sqrt{2} y)]^{-1}$, where $g$ is obtained from the boundary condition $S^{\prime}(0)=-w$. The resulting expression for $\sigma_{\mathrm{NW}}$ is

$$
\sigma_{\mathrm{NW}}=\frac{\sqrt{3}(g+3) g^{2}}{6(1+g)^{3}}-\frac{w}{1+g},
$$

where $g=-(1+1 / \sqrt{2} w)+\sqrt{(1+1 / \sqrt{2} w)^{2}-1}$.

In order to obtain the azimuthal angle $\theta$ field, we have to minimize the energy functional given by Eq. (7). In the bulk, we thus have to solve the Laplace equation $\nabla^{2} \theta=0$ with appropriate anchoring conditions at the boundaries. Let $\boldsymbol{\nu}=\left(-\sin \theta_{0}, \cos \theta_{0}, 0\right)$ now be the local normal to the substrate. Using the approximation $\Sigma(\theta)$ $\sim \sigma_{\mathrm{NW}}+\Sigma_{0}^{\prime \prime}(w) \sin ^{2}\left(\theta-\theta_{0}\right)$ found previously, the nematic director field then satisfies approximately that $K \boldsymbol{\nu} \cdot \nabla \theta+\Sigma_{0}^{\prime \prime} \sin \left[2\left(\theta-\theta_{0}\right)\right]=0$. Note that we may assume strong homeotropic anchoring conditions when $L$ is large compared to the extrapolation length $K / 2 \Sigma_{0}^{\prime \prime} \sim K / w$ or, equivalently, $w L \gg 1$. We can justify this assumption by considering the following rescaling: $\mathbf{r}^{*}=\mathbf{r} / L, \theta^{*}\left(\mathbf{r}^{*}\right)=\theta(\mathbf{r})$, and $\mathcal{F}_{\mathrm{FO}}^{*}=\mathcal{F}_{\mathrm{FO}} / L$. In this rescaled description, we must minimize the FO free energy (7) in the rescaled domain subject to an effective anchoring potential $\Sigma^{*}\left(\theta^{*}\right)=L \Sigma(\theta)$. This means that the dependence on the size of the system can be absorbed into the coefficients of the anchoring potential: $\sigma_{\mathrm{NW}}$ (which does not affect the anchoring conditions) and $\Sigma_{0}^{\prime \prime}$ (which is rescaled by a factor of $L$ ). Strong anchoring condition is then satisfied when the rescaled extrapolation length $K / 2 \Sigma_{0}^{\prime \prime} L \ll 1$, in agreement with our previous estimate.

\section{RESULTS}

\section{A. Numerical results within the LdG model}

We now turn to the evaluation of the distortion contribution in the LdG model We consider values of $\alpha$ between 0 and $\pi / 2,8 \leq L \leq 96$, and $0 \leq w \leq 2$. In order to get the orientational ordering, we numerically minimize the LdG freeenergy functional by using a conjugate-gradient method. The numerical discretization of the continuum problem is performed with a finite element method combined with adaptive meshing in order to resolve the different length scales that may emerge in the problem [34]. The numerical procedure is similar to that used to study the wetting transition by the nematic phase in this geometry [29]. The numerical minimization of the LdG functional $\mathcal{F}_{\mathrm{LdG}}$ shows that the $N^{\perp}$ texture has lower free energy if $\alpha<\pi / 4$ owing to lesser distortion. Conversely, the $N^{l l}$ texture has lower free energy for $\alpha>\pi / 4$. These results are in agreement with previous studies in the literature [8-11]. As a consequence, we observe bistability in a range of values of $\alpha$ around $\pi / 4$. Figure 3 displays some typical textures. We see that on the substrates the nematic orientation is preferentially homeotropic. Inspection of these textures shows that in general there are no disclinations in bulk, except for large $w$ and $\alpha$ around $\pi / 4$, but there is an important distortion on the nematic director field close to the wedges and apexes. On the other hand, when disclinations are observed in bulk [see, for example, Fig. 3(b) or Fig. 3(c)], they are at a distance on the order of $\xi$ from a wedge or apex, which is almost independent of $L$.

The analysis of the calculated equilibrium free energies confirms that the leading-order contribution to the equilibrium free energy per unit area is $\sigma_{\mathrm{NW}}$, which is a further confirmation that there is strong anchoring on the substrates. As a consequence, we may obtain the elastic part of the free energy per cell (i.e., a slice parallel to the $y z$ plane with width along the $x$ axis equal to the substrate period) and unit length in the $z$ direction, $f_{\text {elastic }}$, as $2 L\left(\left.\mathcal{F}_{\mathrm{LdG}}\right|_{\text {eq }} / \mathcal{A}-\sigma_{\mathrm{NW}}\right)$. Numerical evaluation of $f_{\text {elastic }}$ shows a clear dependence on $L$ 

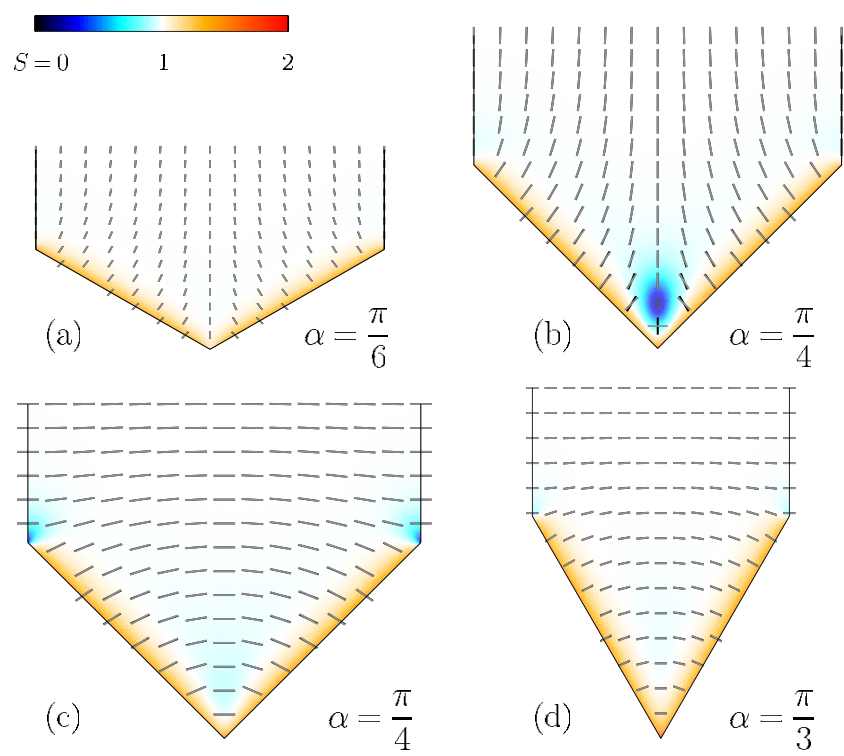

FIG. 3. (Color online) Contour maps of the nematic order parameter $S$ [blue (dark gray) for small $S$, white for the bulk value $S=1$, and orange (light gray) for higher $S$ ] and the nematic director field $\mathbf{n}$ (gray segments) for the equilibrium textures obtained from minimization of the LdG model for $L=16$ and $w=1.0$ : (a) $\alpha=\pi / 6$ $\left(N^{\perp}\right)$, (b) $\alpha=\pi / 4\left(N^{\perp}\right)$, (c) $\alpha=\pi / 4\left(N^{\|}\right)$, and (d) $\alpha=\pi / 3\left(N^{\|}\right)$. The singular nematic director field from the FO approach [Eqs. (11) and (12)] is also shown for comparison (black segments). Note that they are indistinguishable almost everywhere.

in a broad range of values of $w$ (see Fig. 4 for $\alpha=\pi / 6$ and $\alpha=\pi / 3)$. It is interesting to compare this result to the prediction within Berreman's approach for the elastic contribution to a smooth substrate-nematic surface free energy [15]. Although originally this approach was introduced for shallow sinusoidal substrates with strong tangential anchoring, it can be extended straightforwardly to the present case with homeotropic anchoring, either weak or strong. By using the electrostatic analogy, we may expand the azimuthal angle field $\theta$ as



FIG. 4. (Color online) Plot of $f_{\text {elastic }}$ as a function of the anchoring strength $w$ for $\alpha=\pi / 6$ (black symbols) and $\alpha=\pi / 3$ [orange (light gray) symbols]. We considered different cell sizes: $L=8$ (circles), $L=16$ (squares), $L=32$ (diamonds), $L=48$ (up triangle), $L=64$ (left triangle), and $L=96$ (down triangle).

$$
\theta(x, y)=\sum_{n=0}^{\infty} M_{n} \sin (n q x) \exp (-n q y),
$$

where $q$ is the wave number associated with the substrate periodicity, and the coefficients $M_{n}$ are chosen to satisfy the imposed boundary conditions. Substitution of this expression in the FO free energy leads to the elastic contribution to the surface free energy which depends only on $A q$, with $A$ as the roughness amplitude. For sinusoidal substrates under moderate or strong anchoring conditions, Berreman's approximation $f_{\text {elastic }} \approx \pi K(A q)^{2}$ is valid if $q A$ is smaller than 1 [21]. In any case, if $A$ scales like $L$, the surface free energy should be independent of $L$. In order to explain and characterize the anomalous scaling for the elastic free energy obtained within the LdG framework, we will now resort to the FO approximation.

\section{B. Results with the FO model}

Disclination lines are known to nucleate on wedges and apexes [8-11]. Their presence introduces a singular contribution to the orientation field $\theta$ which cannot be expanded as in Eq. (9). We may write $\theta(x, y)$ as $\theta_{s}(x, y)+\theta_{\mathrm{ns}}(x, y)$, where $\theta_{s}$ is the singular contribution due to the disclination lines and $\theta_{\mathrm{ns}}$ is the nonsingular part. Note that Berreman assumed that $\theta_{s} \equiv 0$. The singular $\theta$ field associated with one isolated disclination line located at the origin and characterized by a winding number $I$ takes the form $-I \arctan (x / y)=I \Im[\ln (i \zeta)]$, where $\zeta=x+i y$ and $\mathfrak{I}(\zeta)$ is the imaginary part of $\zeta$. In the bulk, the values of the winding number $I$ are restricted to half-integer values to avoid discontinuities in the nematic director field. For a periodic linear array of disclination lines, we may obtain the singular nematic orientation field from a conformal transformation,

$$
\begin{aligned}
\zeta \mapsto \zeta^{\prime} & =\sin \left(\frac{q \zeta}{2}\right) \\
& =\sin \left(\frac{q x}{2}\right) \cosh \left(\frac{q y}{2}\right)+i \cos \left(\frac{q x}{2}\right) \sinh \left(\frac{q y}{2}\right),
\end{aligned}
$$

where the wave number which characterizes the substrate periodicity is $q=\pi / L \cos \alpha$. The transformation maps a neighborhood of any point $\zeta_{n}=n(2 L \cos \alpha)$, with $n$ as an integer, to a neighborhood of the origin in the $\zeta^{\prime}$-complex plane. If we denote $\zeta=\zeta_{n}+\epsilon(|\epsilon|$ small $)$, then the transformation leads to a complex number $\zeta^{\prime} \approx(-1)^{n} q \epsilon / 2$. Thus, the orientation field $I \Im\left[\ln \left(i \zeta^{\prime}\right)\right]=-I \arctan [\tan (q x / 2) \operatorname{coth}(q y / 2)]$, which is a solution to the Laplace equation, periodic in $x$ with period $2 L \cos \alpha$ and singular at each $\zeta_{n}$, reduces to the field corresponding to an isolated defect of winding number $I$ in the neighborhood of any $\zeta_{n}$. Focusing on the range $x \in[-L \cos \alpha, L \cos \alpha]$, the azimuthal angle generated by this function is $\theta=0$ at $x=0$ and $x= \pm L \cos \alpha$. On the other hand, for $|q y / 2| \gg 1, \quad \theta \approx-\operatorname{sgn}(y) \operatorname{Iqx} / 2$ for $x \neq \pm L \cos \alpha$, where $\operatorname{sgn}(y)=y /|y|$ is the sign of $y$. Note that there is a jump from $\theta=-\operatorname{sgn}(y) I \pi / 2$ to $\theta=\operatorname{sgn}(y) I \pi / 2$ when crossing $x=L \cos \alpha$. In the bulk, this solution is acceptable if $I$ is an 
integer (note that the nematic state is invariant under inversion of the nematic director). If $I$ is a half-integer, we may add to this solution the orientation field $I \arctan [\tan (q x / 2)]$ due to an array of disclination lines with winding number $-I$ located at $\zeta_{n}-i \infty$, which in the $x$ interval $(-L \cos \alpha, L \cos \alpha)$ reduces to $I q x / 2$. The compound orientation field I\{-arctan$[\tan (q x / 2) \operatorname{coth}(q y / 2)]$ $+\arctan [\tan (q x / 2)]\}$ has no discontinuity at $x= \pm L \cos \alpha$ for $y>0$ (in fact, it goes to zero as $q y / 2 \gg 1$ ), but it jumps from $I \pi$ to $-I \pi$ when crossing $x=L \cos \alpha$ for $y<0$. This is again physically acceptable if $I$ is a half-integer. Finally, we must mention that there are different physically equivalent representations of the same singular field associated with a periodic array of disclination lines located at $x=\zeta_{n}$ as, for example, the field $I\{\arctan [\cot (q x / 2) \tanh (q y / 2)]-\arctan [\cot (q x / 2)]\}$. This orientation field shows a physically acceptable discontinuity at $x=0$ for $y<0$ if $I$ is a half-integer.

When disclination lines are located on surfaces, their winding numbers $I$ are not constrained to half-integer values [8-11], as we may tune the winding numbers in order to match the boundary conditions close to any wedge or the apex in the strong anchoring regime. Alternatively, a Schwartz-Christoffel transformation may be used [8]. For the $N^{\perp}$ texture, we find that the winding numbers $I_{1}$ and $I_{2}$ for the disclination lines at the wedge bottom and at the apex top, respectively, are $I_{1}=-\alpha /(\pi / 2-\alpha)$ and $I_{2}=\alpha /(\pi / 2+\alpha)$. For the $N^{\|}$texture, the topological charges are $I_{1}=1$ and $I_{2}=-(\pi / 2-\alpha) /(\pi / 2+\alpha)$. The resulting singular orientation fields, $\theta_{s}^{\perp}$ and $\theta_{s}^{\|}$, can be written in terms of the wave number $q$ and for $x \in(-L \cos \alpha, L \cos \alpha)$ as

$$
\begin{aligned}
\theta_{s}^{\perp}= & \frac{-\alpha}{\frac{\pi}{2}-\alpha}\left[-\arctan \left(\tan \frac{q x}{2} \operatorname{coth} \frac{q y}{2}\right)+\arctan \left(\tan \frac{q x}{2}\right)\right] \\
& +\frac{\alpha}{\frac{\pi}{2}+\alpha}\left\{-\arctan \left[\tan \frac{q x}{2} \tanh \frac{q(y-L \sin \alpha)}{2}\right]\right. \\
& \left.+\arctan \left[\tan \frac{q x}{2}\right]\right\}, \\
\theta_{s}^{\|}= & \frac{\pi}{2}+\left[-\arctan \left(\tan \frac{q x}{2} \operatorname{coth} \frac{q y}{2}\right)+\arctan \left(\tan \frac{q x}{2}\right)\right] \\
& -\frac{\frac{\pi}{2}-\alpha}{\frac{\pi}{2}+\alpha}\left\{-\arctan \left[\tan \frac{q x}{2} \tanh \frac{q(y-L \sin \alpha)}{2}\right]\right. \\
& \left.+\arctan \left[\tan \frac{q x}{2}\right]\right\} .
\end{aligned}
$$

These expressions do not show any discontinuity above the substrate. We checked that these solutions approximate quite well the boundary anchoring conditions (but not exactly), so that $\theta_{\mathrm{ns}}$ may be neglected in most of the cases. Furthermore, the agreement between our Ansätze for the director orientation field and the numerical $\mathrm{LdG}$ textures is excellent except close to the defect cores, as can be seen in Fig. 3.

The elastic energy can be obtained by standard contour integration techniques. By symmetry, $f_{\text {elastic }}$ can be obtained

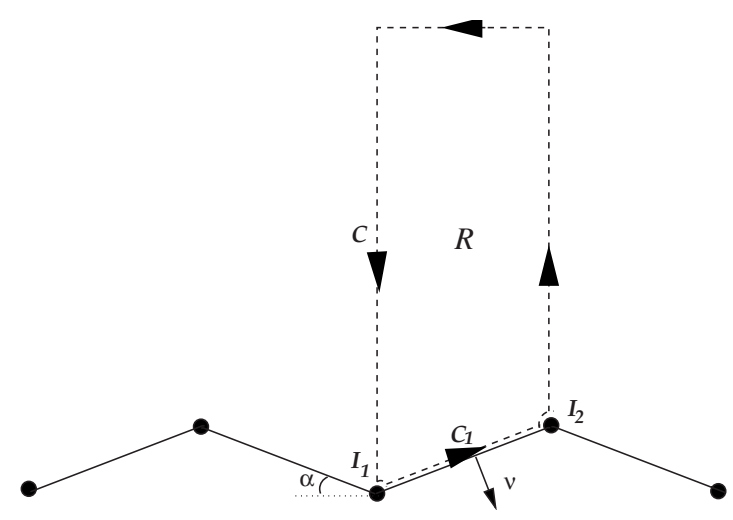

FIG. 5. Plot of the integration contour $C$ (dashed line) to get the elastic contribution to the free energy in the region $R$ inside the contour. The contour is deformed to avoid the cores of the disclination lines with wandering numbers $I_{1}$ and $I_{2}$ at the wedge and apex (filled circles). We highlight the contour $C_{1}$ with an associated outward normal $\boldsymbol{\nu}$

as twice the contribution of the half-cell region $R$ (see Fig. 5). By using the identity $\nabla \cdot(\theta \nabla \theta)=|\nabla \theta|^{2}+\theta \nabla^{2} \theta$, and using the divergence theorem and the fact that $\nabla^{2} \theta=0$ in $R$, we obtain that $f_{\text {elastic }}$ can be expressed in terms of an integral over the contour $C$ of $R$,

$$
f_{\text {elastic }}=K \oint_{C} \theta \boldsymbol{\nu} \cdot \nabla \theta d s \text {. }
$$

Along the segments $x=0, x=L \cos \alpha$, and $y \rightarrow \infty$, we impose that $\theta=\theta_{\text {sing }}=\alpha_{\infty}$, where the far-field azimuthal angle is $\alpha_{\infty}=0$ for the $N^{\perp}$ texture and $\alpha_{\infty}=\pi / 2$ for the $N^{\|}$texture. We link $C_{1}$ to the $x=0$ and $x=L \cos \alpha$ segments by circle arcs of small radii $\epsilon$. These circles take into account the presence of a defect core, and their radii are proportional to the nematic coherence length, which provides a microscopic cutoff for the continuum Frank-Oseen model. In these boundaries, $\theta$ approaches, up to a constant, the field of one isolated disclination line, which satisfies the following property $\boldsymbol{\nu} \perp \nabla \theta$ as $\epsilon \rightarrow 0$. So we can neglect the contribution of the circle arcs to the contour integral. Finally, $\theta=\alpha$ along the segment $C_{1}$ for strong anchoring conditions. Taking into account that $\oint_{C} \boldsymbol{\nu} \cdot \nabla \theta d s=0$, we may rewrite Eq. (13) as

$$
f_{\text {elastic }}=K \oint_{C}\left(\theta-\alpha_{\infty}\right) \nu \cdot \nabla \theta d s=K\left(\alpha-\alpha_{\infty}\right) \oint_{C_{1}} \nu \cdot \nabla \theta d s
$$

If $\theta$ is split into singular and nonsingular contributions, we see that the nonsingular term, which can be expressed by Eq. (9), leads to a contribution to $f_{\text {elastic }}$ independent of $L$. On the other hand, the leading-order contribution to $f_{\text {elastic }}$ comes from the singular orientation field, namely, the contribution close to the disclination lines and apex. If $s$ is the distance of a point of the contour $C_{1}$ close to the wedge or apex, $\boldsymbol{\nu} \cdot \nabla \theta_{\text {sing }} \approx-I_{1} / s$ or $-I_{2} / s$, respectively. The leading-order contribution to $f_{\text {elastic }}$ comes from integration on $C_{1}$ as 


$$
f_{\text {elastic }} \sim \mathcal{K}(\alpha) \ln L / \epsilon,
$$

where $\mathcal{K}$ is defined as

$$
\mathcal{K}(\alpha)=K\left(\alpha-\alpha_{\infty}\right)\left(I_{2}-I_{1}\right)= \begin{cases}\frac{K \pi \alpha^{2}}{\left(\frac{\pi}{2}\right)^{2}-\alpha^{2}}, & \alpha<\frac{\pi}{4} \\ K \pi \frac{\frac{\pi}{2}-\alpha}{\frac{\pi}{2}+\alpha}, & \alpha>\frac{\pi}{4} .\end{cases}
$$

Expressions (15) and (16) show that the equilibrium texture is $N^{\perp}$ for $\alpha<\pi / 4$, and $N^{\|}$for larger values of $\alpha$, in agreement with our LdG calculations and previous results reported in the literature [8].

\section{Analysis of the results within the LdG model}

Finally, in order to check the accuracy of our approximation, we analyze the results obtained with the $\mathrm{LdG}$ model by fitting the values of $f_{\text {elastic }}$ obtained from minimization to an expression

$$
f_{\text {elastic }}=\mathcal{K}(\alpha) \ln (L / \xi)+B(\alpha, w),
$$

where $\mathcal{K}(\alpha)$ is given by expression (16) and $B(\alpha, w)$ is expected not to depend explicitly on $L$ if $w L$ is large enough (i.e., under strong anchoring conditions). The resulting curves for different values of $L$ of $B(\alpha, w)$ as a function of $w$ for a fixed value of $\alpha$ collapse into a master curve (see Fig. $6)$. Deviations only appear for small values of $w \leqq L^{-1}$, at which the strong anchoring condition breaks down.

This result shows clearly that there is a contribution to the elastic part of the surface free energy which scales logarithmically with the periodicity of the substrate. For large $L$ (small $q$ ) and $w \gg L^{-1}$, the surface free-energy density (i.e., per unit projected area on the $x z$ plane) of a nematic in the presence of a sawtooth substrate has the asymptotic behavior $\sigma_{\mathrm{NW}} / \cos \alpha-[\mathcal{K}(\alpha) / 2 \pi] q \ln q+\mathcal{O}(q)$. The nonanalytical contribution $-q \ln q$ introduces a slow decay of the surface free energy of the nematic with increasing $L$. This may help to explain the large deviations with respect to the Wenzel law observed for the wetting transition by nematic of a sawtoothed substrate in contact with the isotropic phase [29].

\section{CONCLUSIONS}

In this paper we have analyzed the size dependence of the elastic contribution to the interfacial free-energy density of a



FIG. 6. (Color online) Plot of $B(w, \alpha)$ as a function of the anchoring strength $w$ for $\alpha=\pi / 6$ and $\alpha=\pi / 3$. The meaning of the symbols is the same as in Fig. 4.

nematic in the presence of a sawtoothed substrate. The nucleation of non-half-integer disclination lines of the nematic director field on the apexes and wedges of the substrate, predicted in the FO model, induces a nonanalytical contribution which scales with the substrate periodicity wave number $q$ as $-q \ln q$ for small $q$. This has been confirmed by the numerical results in the full LdG model. The periodicity scaling of the elastic contribution to the surface free energy is different from that obtained for smooth surfaces, which scales linearly with $q$. Our arguments are not specific to this kind of substrate and can be extended straightforwardly to any surface shape which shows ridges, cusps, and similar singularities. On the other hand, the size scaling of the elastic free energy has consequences for surface transitions such as wetting. Finally, the recent impressive advances in microfluidic technology and surface patterning open the possibility of an experimental verification of our predictions.

\section{ACKNOWLEDGMENTS}

The authors wish to thank Professor M. M. Telo da Gama and Professor A. O. Parry for enlightening discussions. We acknowledge the support from MICINN (Spain) through Grants No. HP2008-0028 and No. FIS2009-09326, Junta de Andalucía (Spain) through Grant No. P09-FQM-4938 (J.M.R.-E.), FCT (Portugal) through Grant No. SFRH/BPD/ 20325/2004 (C.-T.P.), and Acção Integrada Luso-Espanhola Ref. E 17/09 (P.P.).
[1] M. Kléman, Points, Lines and Walls in Liquid Crystals, Magnetic Systems, and Various Disordered Media (Wiley, New York, 1983).

[2] N. D. Mermin, Rev. Mod. Phys. 51, 591 (1979).

[3] M. B. Hindmarsh and T. W. B. Kibble, Rep. Prog. Phys. 58, 477 (1995).

[4] D. J. Kosterlitz and D. J. Thouless, J. Phys. C 6, 1181 (1973).
[5] D. R. Nelson, in Phase Transitions and Critical Phenomena, edited by C. Domb and J. L. Lebowitz (Academic, New York, 1983), Vol. 7, p. 1

[6] H. W. Oseen, Trans. Faraday Soc. 29, 883 (1933).

[7] F. C. Frank, Discuss. Faraday Soc. 25, 19 (1958).

[8] G. Barbero, Lett. Nuovo Cimento Soc. Ital. Fis. 29, 553 (1980) 
[9] G. Barbero, Lett. Nuovo Cimento Soc. Ital. Fis. 32, 60 (1981).

[10] G. Barbero, Lett. Nuovo Cimento Soc. Ital. Fis. 34, 173 (1982).

[11] A. Poniewierski, Eur. Phys. J. E 31, 169 (2010).

[12] L. R. Evangelista and G. Barbero, Phys. Rev. E 50, 2120 (1994).

[13] F. C. Cardoso and L. R. Evangelista, Phys. Rev. E 53, 4202 (1996).

[14] P. V. Dolganov, H. T. Nguyen, E. I. Kats, V. K. Dolganov, and P. Cluzeau, Phys. Rev. E 75, 031706 (2007).

[15] D. W. Berreman, Phys. Rev. Lett. 28, 1683 (1972).

[16] P.-G. de Gennes and J. Prost, The Physics of Liquid Crystals, 2nd ed. (Clarendon Press, Oxford, 1995).

[17] C. V. Brown, M. J. Towler, V. C. Hui, and G. P. Bryan-Brown, Liq. Cryst. 27, 233 (2000).

[18] S. Kitson and A. Geisow, Appl. Phys. Lett. 80, 3635 (2002).

[19] J. I. Fukuda, M. Yoneya, and H. Yokoyama, Phys. Rev. Lett. 98, 187803 (2007).

[20] L. Harnau, S. Kondrat, and A. Poniewierski, Phys. Rev. E 76, 051701 (2007).

[21] G. Barbero, A. S. Gliozzi, M. Scalerandi, and L. R. Evangelista, Phys. Rev. E 77, 051703 (2008).
[22] Y. Yi, G. Lombardo, N. Ashby, R. Barberi, J. E. Maclennan, and N. A. Clark, Phys. Rev. E 79, 041701 (2009).

[23] C. Uche, S. J. Elston, and L. A. Parry-Jones, J. Phys. D: Appl. Phys. 38, 2283 (2005).

[24] C. Uche, S. J. Elston, and L. A. Parry-Jones, Liq. Cryst. 33, 697 (2006)

[25] P. Patrício, M. M. Telo da Gama, and S. Dietrich, Phys. Rev. Lett. 88, 245502 (2002).

[26] D. Andrienko, M. Tasinkevych, P. Patricio, and M. M. Telo da Gama, Phys. Rev. E 69, 021706 (2004).

[27] P. Sheng, Phys. Rev. Lett. 37, 1059 (1976).

[28] P. Sheng, Phys. Rev. A 26, 1610 (1982).

[29] P. Patrício, C.-T. Pham, and J. Romero-Enrique, Eur. Phys. J. E 26, 97 (2008).

[30] F. N. Braun, T. J. Sluckin, and E. Velasco, J. Phys.: Condens. Matter 8, 2741 (1996).

[31] A. Rapini and M. Papoular, J. Phys. (Paris), Colloq. 30, C4 (1969).

[32] K. Binder and P. Hohenberg, Phys. Rev. B 6, 3461 (1972).

[33] J. W. Cahn, J. Chem. Phys. 66, 3667 (1977).

[34] P. Patrício, M. Tasinkevych, and M. M. Telo da Gama, Eur. Phys. J. E 7, 117 (2002). 